\title{
Stability and Mass Parabola in Integrated Nuclear Model
}

\author{
N.Ghahramany ${ }^{*}$, H.Sarafraza, E. Yazdankish \\ Physics Department, Shiraz University, Shiraz, Iran \\ *Corresponding Author: ghahramany@susc.ac.ir
}

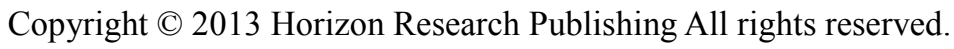

\begin{abstract}
Following our previous introduction of nuclear quark-like model, in this paper, a more precise formula is presented for nuclear binding energy in the context of modified Integrated Nuclear Model (INM). INM is based upon quark-like model with three basic assumptions from which the nuclear binding energy and magic numbers are easily obtained. INM is modified here to give the nuclear mass parabola and most stable nuclei in each nuclei group. Our findings are compared with Liquid Drop Model (LDM), unmodified Integrated Nuclear Model (INM) and experimental data for most stable nuclei.
\end{abstract}

Keywords Stability, Mass Parabola, Integrated Nuclear Model, Binding Energy

\section{Introduction}

Nuclear physicists have been struggling hard for so many years to present a simple and complete nuclear model from which the characteristics of nuclei can be explained and comprehended.The existing successful nuclear models can only explain certain characteristics of the nuclei and make no comments about other nuclear properties. For example Liquid Drop Model (LDM) was presented by Von Weizsacker [1] and then was extended by Bohr and Wheeler [2].This model give the nuclear binding energy formula in terms of $\mathrm{A}$ and $\mathrm{Z}$ [3] as follow:

$$
\begin{array}{r}
B(A, Z)=a_{v} A-a_{s} A^{2 / 3}-a_{c} Z(Z-1) A^{-1 / 3}-a_{a}(N- \\
Z 2 A-1 \pm \delta+\eta
\end{array}
$$

In which five experimentally determined constants are introduced.

Liquid Drop Model (LDM) has been successful in the calculation of binding energy, mass parabola and most stable isobars. However this model fails to predict other properties of nuclei such as, the magic numbers and nuclear magnetic moments. On the other hand Nuclear Shell Model [4] which is based upon Schrodinger equation solution with selected potential such as rounded edge potential well, predict the magic numbers and nuclear magnetic moments by using spin-orbit couplings in a relatively complicated manners $[5,6]$.
We have presented the Integrated Nuclear Model (INM) based upon nuclear quark-like model from which all magic numbers are easily obtained and a new magic number is predicted $[7,8]$. This model is also used to find the magnetic dipole moment of deuteron with greater precision [9]. Using INM, the nuclear binding energy formula was also obtained from quark-like model of nuclei [10] and is given as follow:

$B(A, Z)=\left[A-\left(\frac{\left(N^{2}-Z^{2}\right)+\delta(N-Z)}{3 Z}+3\right)\right] \times \frac{M_{N} c^{2}}{\alpha} \quad A>5$

In formula (2) $\delta(N-Z)= \begin{cases}0, & N \neq Z \\ 1, & N=Z\end{cases}$

and $M_{N} c^{2}$ is the mass of nucleon instead of up-quark mass used in reference [10]. The coefficient $\alpha$ is a dimensionless constant defined to have a range from 90 to 100 [10].

Formula (2) provides the nuclear binding energy for most of the stable nuclei in term of only one coefficient namely $\alpha$ which is simpler than LDM with several coefficients. However this formula needs to be modified to give us the mass parabola in the same way as LDM.

In this paper the modified nuclear binding energy formula is presented in order to find the nuclear mass parabola and the stability of isobaric groups of nuclides. The determination of the mass parabola itself is an indication of the validity of the INM which is based upon the quark structure of the nuclei instead of nucleon structure.

\section{Modified Nuclear Binding Energy Formula in INM}

In the binding energy formula (2) presented in INM a coefficient $\alpha$ is introduced which varies between 90 to 100 for all stable nuclides with $A>5$. The coefficient $\alpha$ may be called "nuclear stability coefficient".

A careful investigation of the stability coefficient for many stable nuclides indicates the fact that $\alpha$ depends upon atomic number $(\mathrm{Z})$ and mass number $(\mathrm{A})$. In fact for isobar nuclides, the stability coefficient $\alpha$ is proportional to atomic number $(Z)$ whereas for isotopic nuclides the stability coefficient $\alpha$ is inversely proportional to the mass number (A). Therefore 


$$
\alpha \propto \frac{Z}{A}
$$

Further analysis of most of the stable nuclides allows proper modification of formula (2) as follow:

$B(A, Z)=$

$\left[A-\left(\frac{\left(N^{2}-Z^{2}\right)+\delta(N-Z)}{3(Z-k)}+3\right)\right] \times \frac{A^{n+s} M_{N} c^{2}}{126(Z-k)} \quad A>5$

In which the coefficient $\mathrm{k}$, $\mathrm{s}$ and $\mathrm{n}$ are defined as

$$
\begin{aligned}
& s=\left\{\begin{aligned}
0.0003, & N, Z \text { even } \\
-0.0003, & N, Z \text { odd }
\end{aligned}\right. \\
& =\left\{\begin{array}{ll}
2, & Z \leq 118 \\
0, & Z>118
\end{array} \quad n=0.87 \text { to } 0.88\right.
\end{aligned}
$$

As can be seen, only one free coefficient namely, $\mathrm{n}$ exist that has a limited range of fine tuning and all other coefficients are known constants.

Now with this modified formula (3), one can find the mass parabolas and stability line.

First we write the nuclear mass as follow:

$$
\begin{aligned}
& M(A, Z)=A M_{N}+\left(M_{H}-M_{N}\right) Z \\
& \quad-\left[A-\left(\frac{\left(N^{2}-Z^{2}\right)+\delta(N-Z)}{3(Z-k)}+3\right)\right] \times \frac{A^{n+s} M_{N}}{126(Z-k)} \\
& =A M_{N}+\left(M_{H}-M_{N}\right) Z-\left[\frac{5}{3} A-\frac{A^{2}+\delta(N-Z)}{3(Z-k)}-3\right] \times \frac{A^{n+s} M_{N}}{126(Z-k)}
\end{aligned}
$$

Differentiating $M(A, Z)$ with respect to $Z$ and equating it to zero give us $Z_{A}$ at which $M(A, Z)$ is minimum. After carrying out the standard calculation, we end up with an equation of power of three in $\mathrm{Z}$ such as:

$$
a Z^{3}+b Z-c=0
$$



(a)
Where

$$
\begin{gathered}
a=\left(M_{H}-M_{N}\right) ; b=\left[\left(\frac{5}{3} A-3\right) \frac{A^{n+s} M_{N}}{126}\right] ; \\
c=\left[\left(\frac{A^{2}+\delta(n-z)}{189}\right) A^{n+s} M_{N}\right]
\end{gathered}
$$

Equation (5) is solved with Maple program and we get one real solution:

$$
Z_{A}=k+\frac{1}{6} \frac{\left[\left[108 c+12 \sqrt{3} \sqrt{\frac{4 b^{3}+27 c^{2} a}{a}}\right] a^{2}\right]^{1 / 3}}{a}
$$

Fig.(1) shows the mass parabolas for odd $\mathrm{A}(A=135)$ and for even $\mathrm{A}(A=102)$ in which $Z_{A}$ is shown by down ward arrows. For odd A with $s=0$ only one parabola is obtained; for even A isobars we get two parabolas.

As shown in fig.(1) for $A=135$ only one stable isobar exist at $Z=56$ which is very close to our finding at $Z_{A}=55.8$ but for even A nuclides depending upon the distance between two obtained parabolas, we get several stable even-even isobars. Here for $A=102$ there are two stable isobars at $Z=44$ and $Z=46$ and for $Z=44$ we get the most stable one, since this is closer to the calculated value namely, $Z_{A}=44.3$. For $\mathrm{LDM}$ for $A=135$, $Z_{A}=55.7$ and for $A=102, Z_{A}=44.7$ [11]. It is seen that our calculated values of $Z_{A}$ is closer to the experimental data as compared to the LDM.

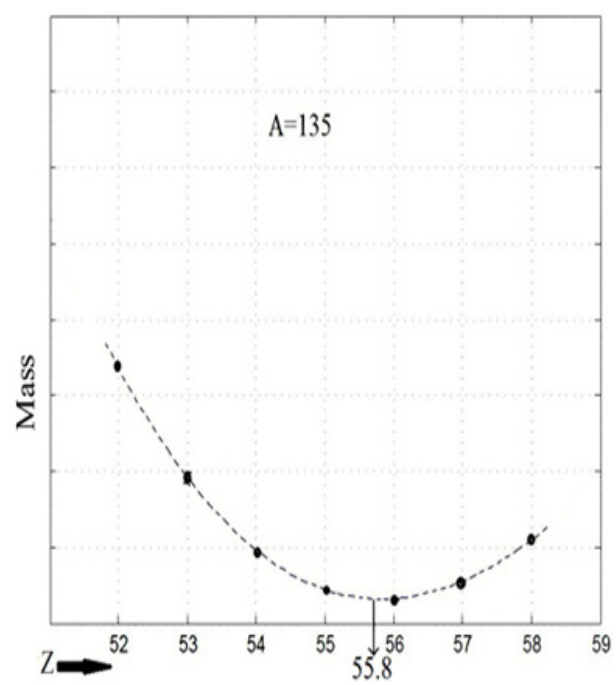

(b)

Figure 1. The isobars mass parabolas a) for even A nuclides b) for odd A nuclides

Using equation (6), we plot $Z_{A}$ verses $\mathrm{N}$ to obtain the stability line shown in figure (2). 


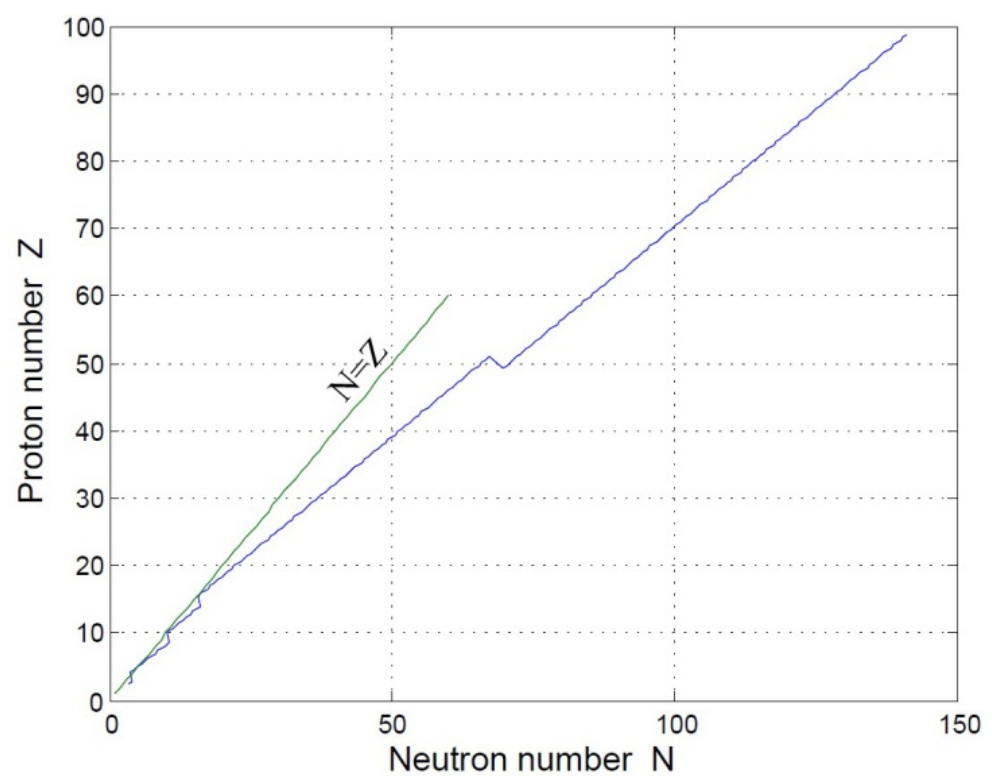

Figure 2. Stability line in modified INM

Finally in table (1) a comparison is made between our findings of nuclear binding energy and the results of other models and experimental data.

\section{Conclusion}

Determination of the stability mass parabola and the nuclear stability line in modified INM and comparison of the most stable nuclei obtained from this model, with the experimental data and LDM is an indication of the validity of the modified INM which is based upon quark-like model of nuclei. Also comparison of nuclear binding energy obtained from modified INM and experimental data, results in a closer match than LDM for most of the light, medium and heavy nuclei. Other characteristics of nuclides are being tested by INM in our research group and the results are promising. 
Table 1. Comparison of nuclear binding energy with different model

\begin{tabular}{|c|c|c|c|c|c|c|}
\hline Z & Nucleus & A & $\begin{array}{c}\mathrm{B}(\mathrm{EXP}) \\
\mathrm{MeV}\end{array}$ & $\begin{array}{c}\mathrm{B}(\mathrm{LDM}) \\
\mathrm{MeV}\end{array}$ & $\begin{array}{c}\mathrm{B}(\mathrm{INM}) \\
\mathrm{MeV}\end{array}$ & $\begin{array}{c}\text { B(Modified INM) } \\
\mathrm{MeV}\end{array}$ \\
\hline 1 & $\mathrm{H}$ & 1 & 0 & -26.461 & & 0 \\
\hline 2 & $\mathrm{He}$ & 4 & 28.296 & 21.9452 & & 28.27305 \\
\hline 3 & $\mathrm{Li}$ & 6 & 31.994 & 27.64 & & 31.7322 \\
\hline 3 & $\mathrm{Li}$ & 7 & 39.244 & 38.3835 & & 38.9815 \\
\hline 4 & $\mathrm{Be}$ & 9 & 58.165 & 56.6316 & 28.27305 & 59.3114 \\
\hline 5 & $\mathrm{~B}$ & 10 & 64.751 & 63.0939 & $\begin{array}{l}30.10864 \\
3258272\end{array}$ & 65.2428 \\
\hline 5 & B & 11 & 76.205 & 75.0627 & 33.58272 & 77.4225 \\
\hline 6 & $\mathrm{C}$ & 12 & 92.162 & 87.749 & 54.71667 & 91.8896 \\
\hline 6 & $\mathrm{C}$ & 13 & 97.108 & 93.629 & 65.03467 & 96.9228 \\
\hline 7 & $\mathrm{~N}$ & 14 & 104.659 & 99.6605 & 75.73481 & 104.2934 \\
\hline 7 & $\mathrm{~N}$ & 15 & 115.492 & 112.2803 & 92.19658 & $\begin{array}{l}104.2934 \\
113.8012\end{array}$ \\
\hline 8 & $\mathrm{O}$ & 16 & 127.619 & 123.7138 & 96.69506 & 127.5033 \\
\hline 8 & $\mathrm{O}$ & 17 & 131.763 & 130.9744 & 104.82993115 .06522 & 130.1135 \\
\hline 8 & $\mathrm{O}$ & 18 & 139.807 & 141.24997 & 127.94649131 .23772 & 138.5735 \\
\hline 9 & $\mathrm{~F}$ & 19 & 147.801 & 149.6775 & 139.15385 & 145.9720 \\
\hline 10 & $\mathrm{Ne}$ & 20 & 160.645 & 160.15493 & 147.91676 & 158.8530 \\
\hline 10 & $\mathrm{Ne}$ & 21 & 167.406 & 168.363 & 160.75488 & 167.4783 \\
\hline 10 & $\mathrm{Ne}$ & 22 & 177.77 & 179.44476 & 167.29278 & $\begin{array}{l}10 / .4 / 03 \\
177.7011\end{array}$ \\
\hline 11 & $\mathrm{Na}$ & 23 & 186.564 & 188.0092 & 178.76377 & 186.7211 \\
\hline 12 & $\mathrm{Mg}$ & 24 & 198.257 & 196.68558 & 186.66229 & \\
\hline 12 & $\mathrm{Mg}$ & 25 & 205.588 & 205.5993 & 198.70651 & 2062926 \\
\hline 12 & $\mathrm{Mg}$ & 26 & 216.681 & 217.2668 & 206.02692 & $\begin{array}{l}200.2920 \\
214.5963\end{array}$ \\
\hline 13 & $\mathrm{Al}$ & 27 & 224.952 & 224.1192 & 217.4098 & $\begin{array}{l}214.5963 \\
2225858\end{array}$ \\
\hline 14 & $\mathrm{Si}$ & 28 & 236.537 & 233.089 & 225.38779 & $\begin{array}{l}222.5858 \\
2371105\end{array}$ \\
\hline 14 & $\mathrm{Si}$ & 29 & 245.011 & 242.5576 & 236.6431 & $\begin{array}{l}237.1105 \\
2467711\end{array}$ \\
\hline 14 & $\mathrm{Si}$ & 30 & 255.62 & 254.6751 & 244.7457 & $\begin{array}{l}246.7711 \\
2557162\end{array}$ \\
\hline 15 & $\mathrm{P}$ & 31 & 262.917 & 260.9052 & 255.17021 & 255.7162 \\
\hline 16 & S & 32 & 271.781 & 269.23215 & 261.40635 & 264.1575 \\
\hline 16 & S & 33 & 280.442 & 279.1541 & 271.82458 & 272.0444 \\
\hline 16 & S & 34 & 291.839 & 291.6321 & 280.5625 & 280.8788 \\
\hline 17 & $\mathrm{Cl}$ & 35 & 298.21 & 29729795 & 292.09649 & 288.9729 \\
\hline 17 & $\mathrm{Cl}$ & 37 & 317.2101 & 317675 & 299.71709 & 297.3346 \\
\hline 18 & $\mathrm{Ar}$ & 36 & 306717 & 30502832 & 317.5582 & 313.3686 \\
\hline 18 & $\mathrm{Ar}$ & 40 & 343811 & 300.02832 & 309.3663 & 304.5650 \\
\hline 10 & K & & 3732724 & & 343.29829 & 343.6705 \\
\hline & & 39 & 333.124 & 333.24958 & 334.60819 & 335.6762 \\
\hline 19 & K & 41 & 351.619 & 354.4661 & 350.2072434690367 & 352.6519 \\
\hline 20 & $\mathrm{Ca}$ & 40 & 342.052 & 340.41858 & 3798567 & 342.5981 \\
\hline 20 & $\mathrm{Ca}$ & 44 & 380.96 & 383.66084 & $\begin{array}{l}3 / 9.856 / 1 \\
3894375\end{array}$ & 382.8547 \\
\hline 21 & $\mathrm{Sc}$ & 45 & 387.848 & 390.6604 & $\begin{array}{c}389.43 / 5 \\
398.22944\end{array}$ & 391.2262 \\
\hline 22 & $\mathrm{Ti}$ & 46 & 398.193 & 399.52474 & & 398.8393 \\
\hline 22 & $\mathrm{Ti}$ & 47 & 407.073 & 408.53494 & 04261420.01354 & 407.5979 \\
\hline 22 & $\mathrm{Ti}$ & 48 & 418.7 & 419.9271 & 426.51645 & 419.2554 \\
\hline 22 & $\mathrm{Ti}$ & 49 & 426.842 & 427.26614 & 437.60839435 .44378 & 427.4059 \\
\hline 22 & $\mathrm{Ti}$ & 50 & 437.781 & 437.02429 & & 436.8867 \\
\hline 23 & $\mathrm{~V}$ & 50 & 434.794 & 434.6673 & & 436.8867 \\
\hline
\end{tabular}

Table (contd.) 


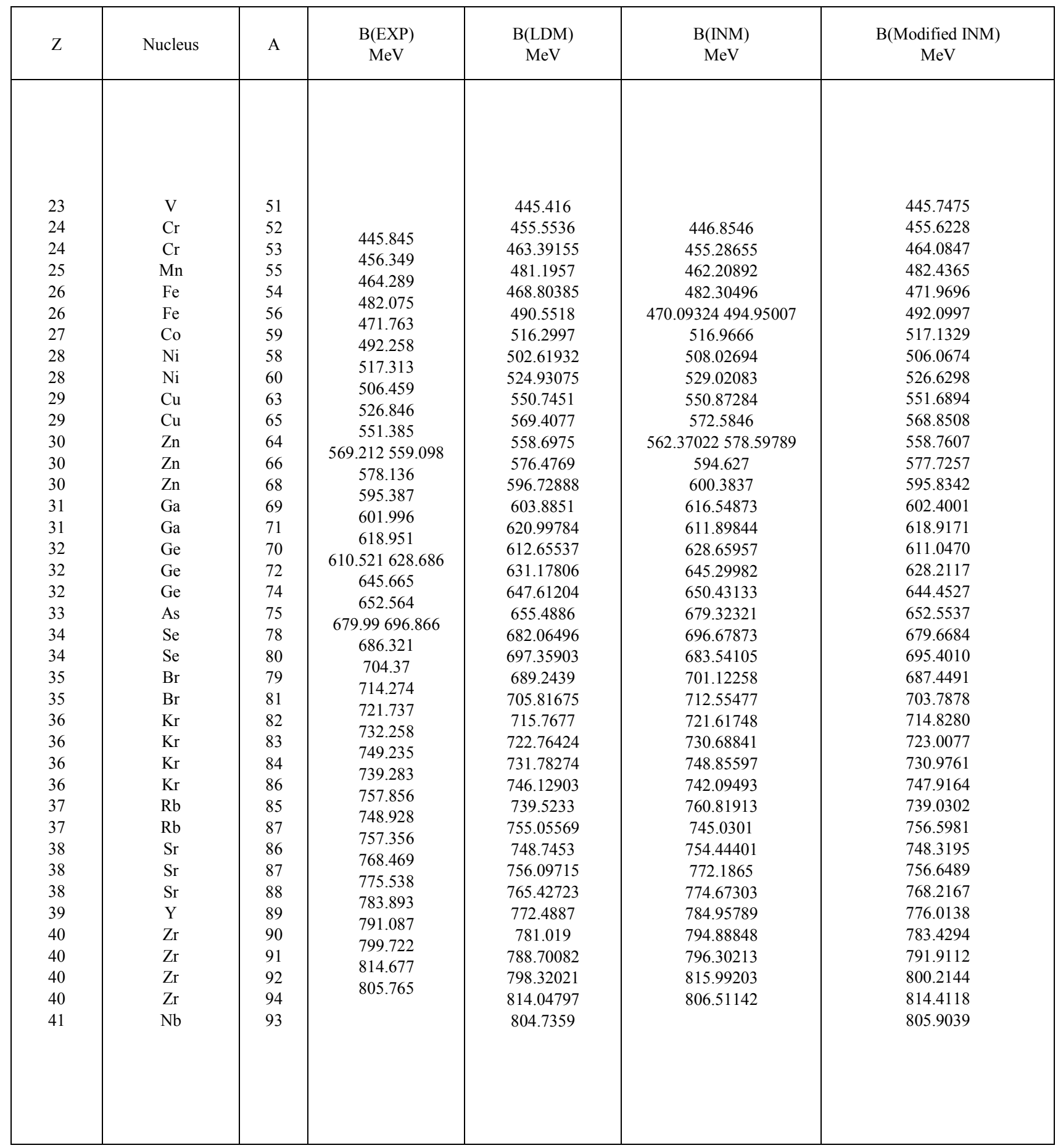

Table (contd.) 


\begin{tabular}{|c|c|c|c|c|c|c|}
\hline $\mathrm{Z}$ & Nucleus & A & $\begin{array}{c}\mathrm{B}(\mathrm{EXP}) \\
\mathrm{MeV}\end{array}$ & $\begin{array}{l}\mathrm{B}(\mathrm{LDM}) \\
\mathrm{MeV}\end{array}$ & $\begin{array}{c}\text { B(INM) } \\
\mathrm{MeV}\end{array}$ & $\begin{array}{c}\text { B(Modified INM) } \\
\text { MeV }\end{array}$ \\
\hline 42 & Mo & 92 & 796.508 & 793.09932 & 795.94785 & 795.4930 \\
\hline 42 & Mo & 95 & 821.625 & 820.5965 & 817.88079 & 821.6087 \\
\hline 42 & Mo & 96 & 830.779 & 830.48545 & 827.97895 & 829.9908 \\
\hline 42 & Mo & 98 & 846.243 & 846.8345 & 848.34648 & 846.2422 \\
\hline 44 & $\mathrm{Ru}$ & 99 & 852.255 & 851.80259 & 857.39063 & 852.8166 \\
\hline 44 & $\mathrm{Ru}$ & 100 & 861.928 & 861.94355 & 858.94508 & 861.2846 \\
\hline 44 & $\mathrm{Ru}$ & 101 & 868.73 & 869.4767 & 869.40782 & 869.5955 \\
\hline 44 & $\mathrm{Ru}$ & 102 & 874.844 & 878.87415 & 870.67943 & 875.7178 \\
\hline 44 & $\mathrm{Ru}$ & 104 & 893.083 & 894.40381 & 891.54317 & 893.5445 \\
\hline 45 & $\mathrm{Rh}$ & 103 & 884.163 & 885.0934 & 880.17114 & 885.1863 \\
\hline 46 & $\mathrm{Pd}$ & 104 & 892.82 & 892.71256 & 889.22905 & 892.3174 \\
\hline 46 & Pd & 105 & 899.914 & 900.53543 & 899.97871 & 900.7118 \\
\hline 46 & $\mathrm{Pd}$ & 106 & 909.474 & 910.189 & 910.81159 & 908.9562 \\
\hline 46 & $\mathrm{Pd}$ & 108 & 925.239 & 926.29915 & 922.81499 & 924.9800 \\
\hline 46 & $\mathrm{Pd}$ & 110 & 940.207 & 941.11778 & 944.64934 & 940.3585 \\
\hline 47 & $\mathrm{Ag}$ & 107 & 915.263 & 915.80492 & 919.77497 & 916.1749 \\
\hline 47 & $\mathrm{Ag}$ & 109 & 931.727 & 932.86781 & 932.11781 & 932.5995 \\
\hline 48 & $\mathrm{Cd}$ & 110 & 940.646 & 940.7986 & 940.98553 & 939.9041 \\
\hline 48 & $\mathrm{Cd}$ & 111 & 947.622 & 948.22807 & 952.19342 & 948.0861 \\
\hline 48 & $\mathrm{Cd}$ & 112 & 957.016 & 957.45559 & 953.35906 & 956.1212 \\
\hline 48 & $\mathrm{Cd}$ & 113 & 963.556 & 964.25463 & 964.54063 & 964.0060 \\
\hline 48 & $\mathrm{Cd}$ & 114 & 972.599 & 972.84985 & 975.82258 & 971.7369 \\
\hline 49 & In & 113 & 963.094 & 963.41231 & 962.12798 & 963.5100 \\
\hline 49 & In & 115 & 979.404 & 979.7077 & 984.90679 & 979.5262 \\
\hline 50 & $\mathrm{Sn}$ & 116 & 988.684 & 987.89353 & 983.20639 & 986.9973 \\
\hline 50 & $\mathrm{Sn}$ & 118 & 1004.955 & 1003.8319 & 1006.25447 & 1005.2137 \\
\hline 50 & Sn & 120 & 1020.546 & 1018.5973 & 1018.68817 & 1020.0715 \\
\hline 51 & $\mathrm{Sb}$ & 121 & 1026.325 & 1025.6927 & 1027.54763 & 1027.0924 \\
\hline 51 & $\mathrm{Sb}$ & 123 & 1042.097 & 1040.1801 & 1040.04681 & 1043.4808 \\
\hline 52 & $\mathrm{Te}$ & 126 & 1066.369 & 1063.5988 & 1061.3598 & 1066.7824 \\
\hline 52 & $\mathrm{Te}$ & 128 & 1081.439 & 1076.7653 & 1085.47929 & 1082.4686 \\
\hline 52 & $\mathrm{Te}$ & 130 & 1095.941 & 1088.937 & 1097.80741 & 1097.6144 \\
\hline 53 & I & 127 & 1072.577 & 1070.8771 & 1069.98274 & 1073.9530 \\
\hline 54 & $\mathrm{Xe}$ & 129 & 1087.651 & 1085.9613 & 1090.45272 & 1089.1120 \\
\hline 54 & $\mathrm{Xe}$ & 131 & 1103.512 & 1100.1915 & 1103.42321 & 1105.2131 \\
\hline 54 & $\mathrm{Xe}$ & 132 & 1112.448 & 1107.9009 & 1115.85749 & 1113.0778 \\
\hline 55 & $\mathrm{Cs}$ & 133 & 1118.528 & 1115.3434 & 1124.19407 & 1117.6565 \\
\hline 56 & $\mathrm{Ba}$ & 137 & 1149.681 & 1144.0675 & 1145.90412 & 1148.4568 \\
\hline 56 & $\mathrm{Ba}$ & 138 & 1158.293 & 1151.5421 & 1158.66304 & 1158.9851 \\
\hline
\end{tabular}

Table (contd.) 


\begin{tabular}{|c|c|c|c|c|c|c|}
\hline $\mathrm{Z}$ & Nucleus & A & $\begin{array}{c}\mathrm{B}(\mathrm{EXP}) \\
\mathrm{MeV}\end{array}$ & $\begin{array}{c}\mathrm{B}(\mathrm{LDM}) \\
\mathrm{MeV}\end{array}$ & $\begin{array}{c}\text { B(INM) } \\
\mathrm{MeV}\end{array}$ & $\begin{array}{c}\text { B(Modified INM) } \\
\mathrm{MeV}\end{array}$ \\
\hline 57 & $\mathrm{La}$ & 138 & 1155.774 & 1151.2653 & 1153.85554 & 1155.7274 \\
\hline 57 & $\mathrm{La}$ & 139 & 1164.551 & 1159.105 & 1166.73445 & 1136.5646 \\
\hline 58 & $\mathrm{Ce}$ & 140 & 1172.692 & 1167.8856 & 1174.39252 & 1170.7473 \\
\hline 58 & $\mathrm{Ce}$ & 142 & 1185.29 & 1181.675 & 1187.94784 & 1186.3789 \\
\hline 59 & $\operatorname{Pr}$ & 141 & 1177.919 & 1174.1085 & 1181.66244 & 1177.7014 \\
\hline 60 & $\mathrm{Nd}$ & 142 & 1185.142 & 1181.5574 & 1188.56759 & 1184.4452 \\
\hline 60 & $\mathrm{Nd}$ & 143 & 1191.266 & 1188.3505 & 1189.38183 & 1192.5988 \\
\hline 60 & $\mathrm{Nd}$ & 144 & 1199.083 & 1196.7746 & 1202.61474 & 1200.6467 \\
\hline 60 & $\mathrm{Nd}$ & 146 & 1212.403 & 1211.0565 & 1216.51726 & 1213.3904 \\
\hline 62 & $\mathrm{Sm}$ & 152 & 1253.104 & 1253.5745 & 1258.49943 & 1252.2646 \\
\hline 62 & $\mathrm{Sm}$ & 154 & 1266.94 & 1266.5934 & 1272.46549 & 1267.1604 \\
\hline 63 & $\mathrm{Eu}$ & 151 & 1244.141 & 1245.4718 & 1250.92515 & 1243.8002 \\
\hline 63 & $\mathrm{Eu}$ & 153 & 1258.998 & 1260.0164 & 1252.0625 & 1259.4052 \\
\hline 64 & $\mathrm{Gd}$ & 156 & 1281.598 & 1281.9731 & 1286.04737 & 1281.7931 \\
\hline 64 & $\mathrm{Gd}$ & 158 & 1295.896 & 1295.468 & 1300.35239 & 1296.8405 \\
\hline 64 & $\mathrm{Gd}$ & 160 & 1309.29 & 1308.1556 & 1314.5448 & 1308.1365 \\
\hline 65 & $\mathrm{~Tb}$ & 159 & 1302.027 & 1301.9809 & 1306.82008 & 1300.7987 \\
\hline 66 & Dy & 162 & 1324.106 & 1323.6067 & 1327.56172 & 1322.9576 \\
\hline 66 & Dy & 163 & 1330.377 & 1329.4064 & 1327.81106 & 1330.3749 \\
\hline 66 & Dy & 164 & 1338.035 & 1336.7617 & 1342.0879 & 1337.6889 \\
\hline 67 & Ho & 165 & 1344.256 & 1343.3234 & 1348.27368 & 1345.0398 \\
\hline 68 & Er & 166 & 1351.572 & 1351.0262 & 1354.12255 & 1352.1740 \\
\hline 68 & Er & 167 & 1358.008 & 1357.0604 & 1354.45782 & 1359.0660 \\
\hline 68 & Er & 168 & 1365.779 & 1364.6314 & 1368.95728 & 1367.0472 \\
\hline 69 & $\mathrm{Tm}$ & 169 & 1371.352 & 1370.6194 & 1374.66657 & 1370.7159 \\
\hline 70 & $\mathrm{Yb}$ & 172 & 1392.764 & 1391.7813 & 1395.18194 & 1392.6360 \\
\hline 70 & $\mathrm{Yb}$ & 173 & 1399.131 & 1397.6674 & 1395.41458 & 1399.9972 \\
\hline 70 & $\mathrm{Yb}$ & 174 & 1406.595 & 1405.069 & 1410.24421 & 1407.2110 \\
\hline 71 & $\mathrm{Lu}$ & 175 & 1412.106 & 1411.0893 & 1415.66989 & 1410.8362 \\
\hline 71 & $\mathrm{Lu}$ & 176 & 1418.394 & 1416.9087 & 1415.85338 & 1418.0879 \\
\hline 72 & $\mathrm{Hf}$ & 177 & 1425.185 & 1424.3349 & 1421.10223 & 1425.2612 \\
\hline 72 & Hf & 178 & 1432.811 & 1431.9414 & 1436.13156 & 1431.4239 \\
\hline 72 & Hf & 180 & 1446.297 & 1444.9382 & 1451.43158 & 1446.5547 \\
\hline 73 & $\mathrm{Ta}$ & 181 & 1452.24 & 1450.9735 & 1456.56802 & 1453.2859 \\
\hline 74 & W & 182 & 1459.335 & 1458.1089 & 1461.40522 & 1459.8504 \\
\hline 74 & W & 183 & 1465.525 & 1464.0784 & 1461.62301 & 1464.6875 \\
\hline 74 & W & 184 & 1472.937 & 1471.526 & 1476.98029 & 1471.2362 \\
\hline 75 & $\mathrm{Re}$ & 185 & 1478.341 & 1477.0087 & 1481.67469 & 1477.8280 \\
\hline 75 & $\mathrm{Re}$ & 187 & 1491.877 & 1490.2904 & 1497.36935 & 1492.8904 \\
\hline
\end{tabular}

Table (contd.) 


\begin{tabular}{|c|c|c|c|c|c|c|}
\hline $\mathrm{Z}$ & Nucleus & A & $\begin{array}{c}\text { B(EXP) } \\
\mathrm{MeV}\end{array}$ & $\begin{array}{c}\mathrm{B}(\mathrm{LDM}) \\
\mathrm{MeV}\end{array}$ & $\begin{array}{c}\text { B(INM) } \\
\text { MeV }\end{array}$ & $\begin{array}{c}\text { B(Modified INM) } \\
\text { MeV }\end{array}$ \\
\hline 76 & Os & 189 & 1505.007 & 1503.2513 & 1502.04693 & 1503.9947 \\
\hline 76 & Os & 190 & 1512.799 & 1510.5523 & 1517.73611 & 1510.5094 \\
\hline 76 & Os & 192 & 1526.116 & 1523.036 & 1533.53906 & 1525.0828 \\
\hline 77 & Ir & 191 & 1518.088 & 1516.0377 & 1522.14121 & 1517.1743 \\
\hline 77 & Ir & 193 & 1532.058 & 1529.0561 & 1538.08144 & 1531.9626 \\
\hline 78 & $\mathrm{Pt}$ & 194 & 1539.577 & 1536.145 & 1542.34012 & 1538.6738 \\
\hline 78 & $\mathrm{Pt}$ & 195 & 1545.682 & 1541.8699 & 1542.38144 & 1546.0526 \\
\hline 78 & $\mathrm{Pt}$ & 196 & 1553.604 & 1549.0352 & 1558.40616 & 1553.3518 \\
\hline 79 & $\mathrm{Au}$ & 197 & 1559.386 & 1554.5095 & 1562.51729 & 1560.1086 \\
\hline 80 & $\mathrm{Hg}$ & 198 & 1566.489 & 1561.0472 & 1566.36429 & 1566.7061 \\
\hline 80 & $\mathrm{Hg}$ & 199 & 1573.153 & 1566.9765 & 1566.48393 & 1574.1315 \\
\hline 80 & $\mathrm{Hg}$ & 200 & 1581.181 & 1574.3316 & 1582.67354 & 1581.4803 \\
\hline 80 & $\mathrm{Hg}$ & 201 & 1587.411 & 1579.9487 & 1582.63325 & 1588.7515 \\
\hline 80 & $\mathrm{Hg}$ & 202 & 1595.165 & 1586.9882 & 1598.99688 & 1595.9441 \\
\hline 81 & $\mathrm{Tl}$ & 203 & 1600.87 & 1592.4387 & 1602.80964 & 1602.7903 \\
\hline 81 & $\mathrm{Tl}$ & 205 & 1615.072 & 1604.989 & 1619.26432 & 1617.1500 \\
\hline 82 & $\mathrm{~Pb}$ & 206 & 1622.325 & 1611.9829 & 1622.92633 & 1624.0399 \\
\hline 82 & $\mathrm{~Pb}$ & 207 & 1629.063 & 1617.5005 & 1622.8084 & 1631.2074 \\
\hline 82 & $\mathrm{~Pb}$ & 208 & 1636.431 & 1624.4233 & 1639.51406 & 1638.2975 \\
\hline 83 & $\mathrm{Bi}$ & 209 & 1640.23 & 1629.8385 & 1643.0243 & 1640.8417 \\
\hline 90 & Th & 232 & 1766.687 & 1769.1834 & 1764.19153 & 1767.0470 \\
\hline 92 & $\mathrm{U}$ & 235 & 1783.864 & 1785.8988 & 1786.7108 & 1782.1712 \\
\hline 92 & $\mathrm{U}$ & 238 & 1801.69 & 1804.1704 & 1803.59006 & 1802.6499 \\
\hline
\end{tabular}

\section{REFERENCES}

[1] C.F. von Weizsacker, Z. Physik 96, 431 (1935).

[2] N. Bohr and J. A. Wheeler, Phys. Rev. 56, 426 (1939).

[3] H. A. Bethe, Rev. Mod. Phys. 8, 82 (1936).

[4] W. Elsasser, J. phys. Radium 4,549 (1933).

[5] Maria Goeppert-Mayer,Phys.Rev.75:1969-1970(1949).

[6] O.Haxel, J.D.H.Jensen, H.E.Suess,Z.Phys.128,295(1950).

[7] N.Ghahramani,H.Hora,G.H.Miley,M.Ghanaatian,M.Hooshmand,K.Philberth,F.Osman, Phys.Essays 21,200(2008).

[8] N.Ghahramani,M.Ghanaatian,M.Hooshmand,Iranian Physical Journal,vol.1,no.2(2007).

[9] N. Ghahramany and E. Yazdankish. Communication in theoretical physics, Vol.59, No. 5 (2013).

[10] N.Ghahramany,Sh.Gharaati,andM.Ghanaatian,New Approach to Nuclear Binding Energy in Integrated Nuclear Model, Physics of Particles and Nuclei Letters, Vol. 8, No. 2, pp. 97-106(2011).

[11] W. E. Meyerhof, Elements of Nuclear Physics, McGraw-Hill Series in Fundamentals of Phys.( 1967). 